\title{
Experimentos para a Feira de Ciências mediados pelo Diagrama V
}

\author{
Xavier, L. $A^{, 1,2^{\star}}$; Segatto, B. $R^{2} ;$ Ferracioli, L. ${ }^{2}$ \\ 1 Escola Professora Filomena Quitiba, SEDU, Piúma, ES, Brasil. \\ 2 Programa de Pós-Graduação em Ensino de Física (PPGEnFis), Universidade Federal do Espírito Santo UFES, \\ Vitória, ES, Brasil. \\ *e-mail: lucas.perobas@gmail.com
}

\begin{abstract}
Resumo
Este artigo se propõe apresentar os resultados do uso do Diagrama $V$ na Feira de Ciências, que representa ambiente de aprendizagem para o educando. A produção dos diagramas ocorreu em 2018 na escola professora Filomena Quitiba, localizada no município de Piúma/ES. Foram 10 grupos que fizeram uso da heurística desenvolvida por David Bob Gowin [1]. A inserção do diagrama com seus elementos constitutivos se deu por meio de oficinas pedagógicas, ressignificação o método científico. As análises e quantificação dos diagramas foram adotados os critérios de Gowin e Alvarez [2] que oscila conforme o item do instrumento heurístico. O uso do recurso heurístico norteador nas atividades experimentais do evento científico da escola pelos membros dos grupos pode-se inferir resultados positivos, pois houve a percepção da relevância do método científico mimetizado no formato do Diagrama V.
\end{abstract}

Palavras-chaves: Método científico, Diagrama V, Feira de Ciências.

\begin{abstract}
This article proposes to present the results of the use of Diagram $\mathrm{V}$ in the Science Fair, which represents learning environment for the student. The production of the diagrams occurred in 2018 at the school "Professora Filomena Quitiba", located in the municipality of Piúma / ES. There were 10 groups that made use of the heuristic developed by David Bob Gowin [1]. The insertion of the diagram with its constituent elements occurred through pedagogical workshops, re-signification of the scientific method. The analysis and quantification of the diagrams were adopted according to the criteria of Gowin and Alvarez [2] that oscillates according to the item of the heuristic instrument. The use of the heuristic guiding resource in the experimental activities of the scientific event of the school by the members of the groups can be inferred positive results, because there was perception of the relevance of the scientific method mimetizado in the format of the Diagram V.
\end{abstract}

Keywords: Scientific method, Diagram V, Science Fair

\section{Introdução}

O artigo traz os resultados obtidos em 2018 na Feira de Ciências desenvolvido juntamente com 10 grupos de alunos da educação básica. $O$ projeto Feira de Ciências completa este ano 36 anos, promovido pela escola professora Filomena Quitiba. Na busca constante por inovação, foi inserido em 2018 o Diagrama V, instrumento propício para planificar e acompanhar projetos como os desenvolvidos para o evento da escola. A Feira de Ciências oportuniza o educando conhecimento em espaço não formal. São consideradas como eventos sociais, científicos e culturais realizados nas escolas ou na comunidade com a intenção de, durante a apresentação dos estudantes, oportunizar um diálogo com os visitantes, constituindo-se na oportunidade de discussão sobre os conhecimentos, metodologias de pesquisa e criatividade dos alunos em todos os aspectos referentes à exibição de trabalhos [3].

A Figura 1 ilustra o Diagrama $V$ [4], contém elementos para maior entendimento do método científico pelo educando. 


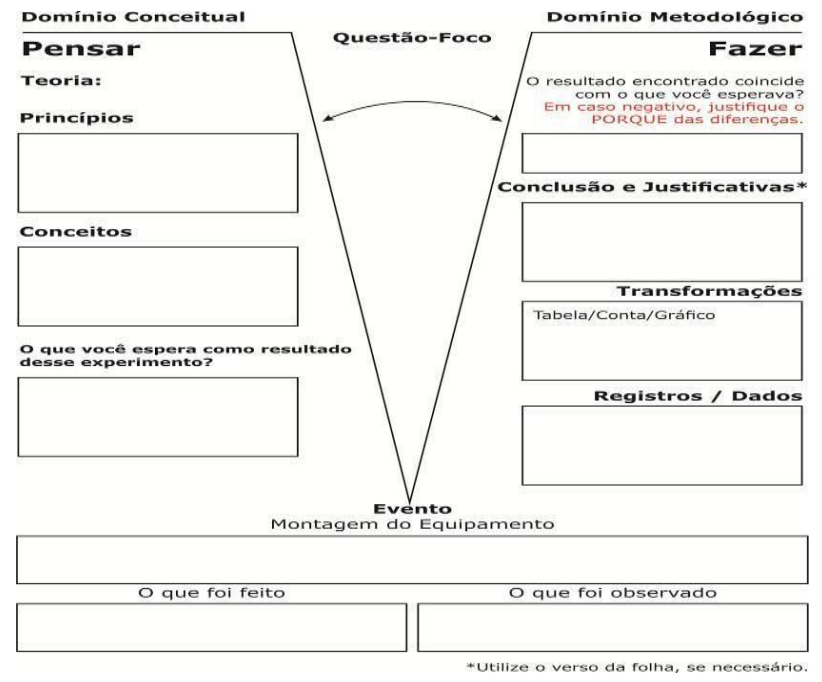

Figura 1: Esquema da organização do Diagrama V (adaptação de Prado, 2015) para o trabalho de investigação.

Afim de popularizar o método científico no evento cientifico que ocorre anualmente na escola se propõe a infusão da heurística de Gowin. Denominada aqui como Diagrama $V$ na preparação dos educandos para os projetos experimentais. o Diagrama $\mathrm{V}$ é útil porque mostra claramente a produção de conhecimentos como resultante da interação entre dois domínios, um teórico-conceitual e outro metodológico [5].

$\mathrm{Na}$ perspectiva de inovações, introduzir a metodologia de trabalho no evento científico requer que o aluno, além de adquirir determinadas informações e desenvolver habilidades para realizar certas tarefas, deve aprender a aprender, para continuar aprendendo [6].

A utilização do Diagrama $V$ com os alunos objetiva obter melhor estratégia de aprendizagem científica e alavancar a qualidade e explicitação dos projetos dos grupos de alunos para a comunidade. E a importância das Feiras de Ciências como local de divulgação científica é despertar o interesse pela ciência justificando a hipótese do ensino e aprendizagem em espaços não formais [7].

\section{Materiais e métodos}

A pesquisa tem um enfoque quali-quantitativo do tipo descritiva baseada na concepção de que a problemática pode ser resolvida e as prática pedagógica pode ser melhorada.
A pesquisa foi desenvolvida na rede pública de ensino, Escola Estadual de Ensino Fundamental e Médio Professora Filomena Quitiba localizada no município de Piúma/ES. Os sujeitos da pesquisa foram 78 alunos das três séries do ensino médio, com predominância do sexo feminino nos grupos formados e com faixa etária de 15 a 17 anos de idade. O trabalho foi desenvolvido em cumprimento à exigência do curso do Mestrado Nacional Profissional em Ensino de Física - MNPEF vinculado ao Mestrado em Ensino de Física do PPGEnsFis da UFES - ES. Para coleta de dados foram utilizados como instrumentos o Diagrama V [4].

Foram realizadas uma oficina sobre o método científico e outra sobre o Diagrama $\mathrm{V}$, realizada com exposição oral em um tempo de aula de 55 minutos, com a utilização de Datashow. Posteriormente foram marcados os encontros organizados para o preenchimento do diagrama.

A etapa final foi o compartilhamento dos diagramas referentes aos trabalhos ilustrados na Tabela 1, junto com a explicitação dos experimentos no dia da Feira de Ciências momento em que cada grupo, apresentou os conceitos aprendidos.

Tabela 1: Temas dos 10 trabalhos estruturados para avaliação

\begin{tabular}{|c|c|}
\hline Grupos & $\begin{array}{c}\text { Trabalhos mediados pelo } \\
\text { Diagrama V }\end{array}$ \\
\hline 1 & $\begin{array}{l}\text { Pressão, pressão atmosférica } \\
\text { e densidade }\end{array}$ \\
\hline 2 & $\begin{array}{l}\text { Prensa hidráulica } \\
\text { multiplicadora de força de } \\
\text { Pascal }\end{array}$ \\
\hline 3 & $\begin{array}{l}\text { Vasos comunicantes para } \\
\text { determinar a densidade de } \\
\text { substâncias }\end{array}$ \\
\hline 4 & $\begin{array}{l}\text { O Teorema de Arquimedes - } \\
\text { empuxo }\end{array}$ \\
\hline 5 & Stand Up ecológico \\
\hline 6 & $\begin{array}{l}\text { O Teorema Bernoulli e tubo } \\
\text { de Venturi }\end{array}$ \\
\hline 7 & $\begin{array}{l}\text { Futebol: um esporte } \\
\text { reduzindo as desigualdades }\end{array}$ \\
\hline 8 & $\begin{array}{l}\text { Primeira Lei de } \text { Ohm em } \\
\text { operação }\end{array}$ \\
\hline 9 & $\begin{array}{l}\text { Circuito série e paralelo: uma } \\
\text { abordagem experimental }\end{array}$ \\
\hline 10 & $\begin{array}{l}\text { A Física no Enem: Fontes de } \\
\text { geração de energia elétrica }\end{array}$ \\
\hline
\end{tabular}

Fonte: Adaptação dos autores, 2018 


\section{Blucher Proceedings \\ X Encontro Científico de Física Aplicada}

\section{Resultados e Discussões}

O trabalho abordou o uso do Diagrama $\mathrm{V}$ nos projetos submetidos ao evento científico da escola, foi adotada a estratégia de oficinas pedagógicas para a inserção do diagrama e dos seus elementos constitutivos. Esta metodologia representa oportunidade para aprender o processo de sistematização do conhecimento científico na prática. Uma boa porção de conhecimento deverá incluir todos os elementos do "Vê", ilustrar como é que esses elementos se ligam entre si, e ser coerente, compreensiva e significativa [8].

São analisados 10 histogramas pontuando os elementos constitutivo do Diagrama $\mathrm{V}$ relacionado aos critérios da escala de Gowin e Alvarez [2]. Primeiramente, no Gráfico 1 é apresentado os resultados para o elemento Questão-Foco. Dos 10 grupos, somente um não conseguiu elaborar este item, três grupos conseguiram elaborá-la, mas não inclui o Evento ou o lado Conceitual do V. Os demais grupos apresentaram, incluindo conceitos para serem usados e diretamente relacionados com o Evento.

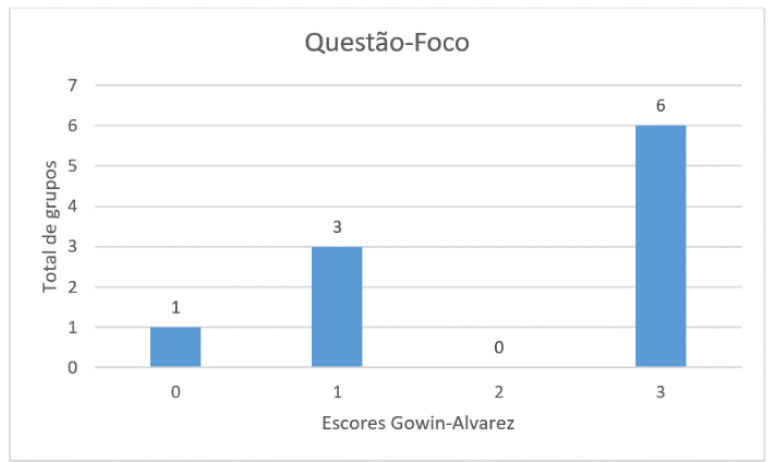

Gráfico 1: Questão-Foco dos diagramas. Fonte: Os autores, 2018

O item Teoria do Diagrama V, cinco grupos tiveram mais facilidade (Gráfico 2). O grupo que apresentou o 'Teorema de Arquimedes - Empuxo' colocou como Hidrostática e Hidrodinâmica, poderia ter colocado somente a primeira, ou simplesmente, Fluidos. Outro grupo 'Stand Up ecológico', também teve dificuldade de relacionar a atividade experimental com a Teoria, foi preciso ajuda do orientador. Um terceiro grupo 'Circuito série e paralelo: uma abordagem experimental, disse que a Teoria era 0 Eletromagnetismo, o mais correto seria Eletrodinâmica.
Um quarto grupo foi certeiro na Teoria, mas sem relação com a Questão-Foco e o Evento.

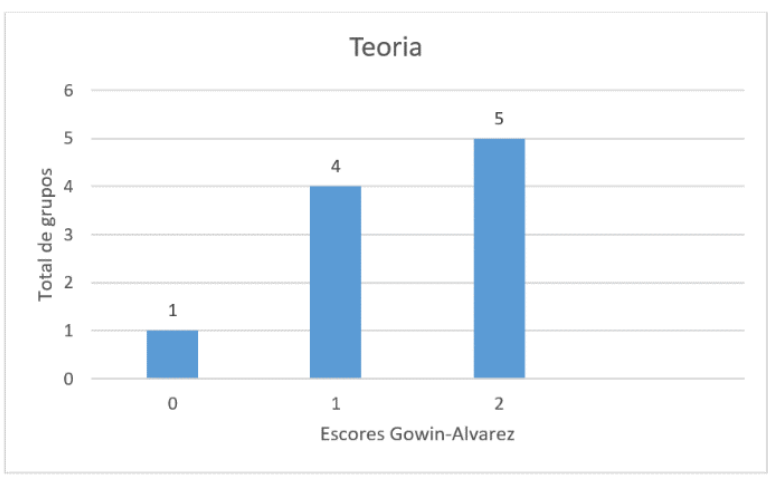

Gráfico 2: Teoria nos diagramas

Fonte: Os autores, 2018

O Gráfico 3, mostra que dois grupos, nenhum Princípio ou Lei são identificados. Os demais grupos foram certeiros neste item.

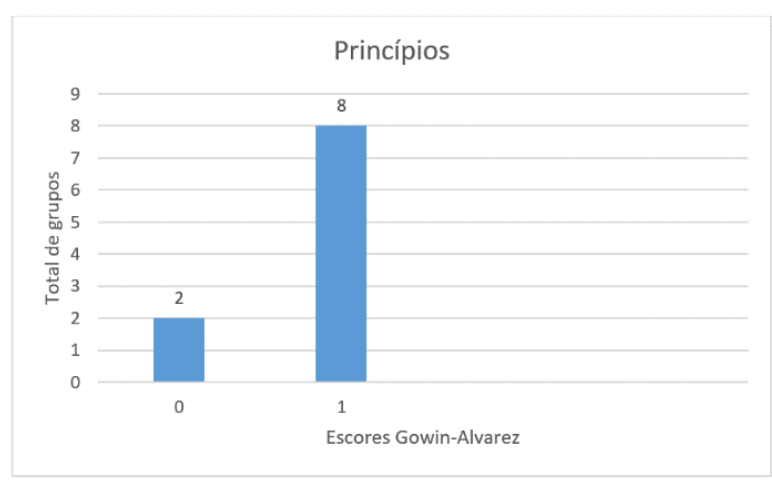

Gráfico 3: Princípios nos diagramas

Fonte: Os autores, 2018

O item Conceitos, retratado no Gráfico 4, mostra que dois grupos apresentam, mas sem relação com a Questão-foco e/ou os Evento. O quinto grupo 'Stand Up ecológico', nenhum Conceito é identificado, não tiveram a preocupação com a parte teórica, ou seja, a Física do experimento. Durante a feira os integrantes desse grupo tiveram a percepção da importância da preparação do trabalho e de seguir um método. Outro grupo conseguiu identificar os Conceitos, mas não estão relacionados com a Questão-foco e/ou os Eventos. 


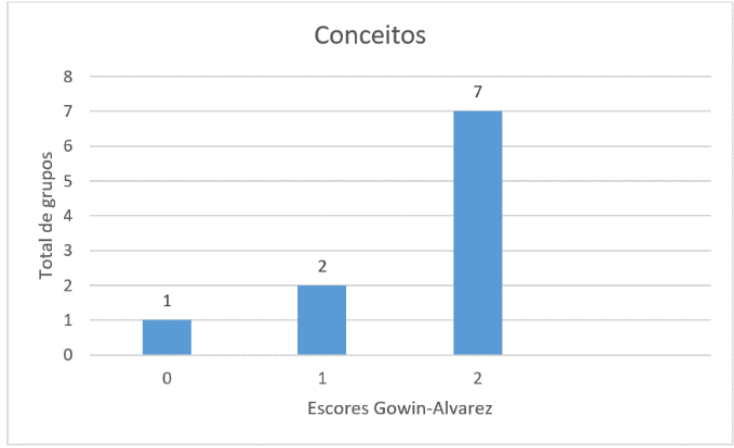

Gráfico 4: Conceitos nos diagramas Fonte: Os autores, 2018

O item $O$ que você Espera como Resultado deste Experimento? não consta na obra de Gowin e Alvarez [2], foi importado [4] para poder realizar a avaliação. O Gráfico 5 mostra que, seis grupos conseguiram apontar expectativas do experimento, relacionadas com a Questão-foco e/ou ao Evento, para os demais grupos, as expectativas são identificadas, mas sem relação com a Questão-foco e/ou ao Evento.

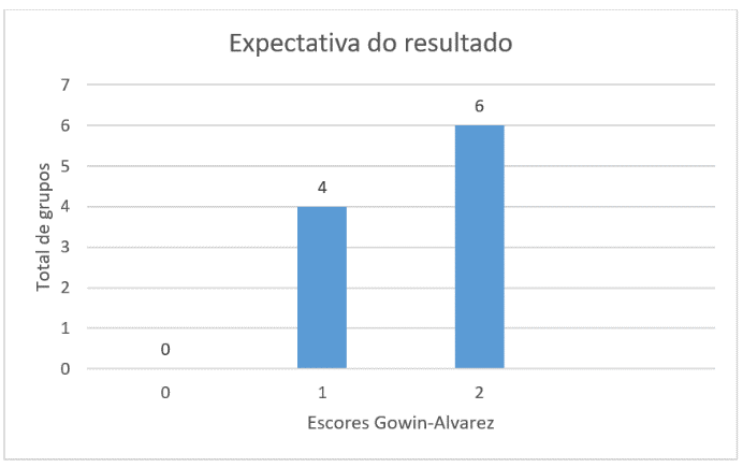

Gráfico 5: Espectativa do resultado do experimento Fonte: Os autores, 2018

O Gráfico 6 é representado com informações sobre o Evento, dois grupos conseguem identificá-lo, mas é inconsistente com a Questão-foco. Oito grupos conseguiram fazer o Evento e relacioná-lo com Questão-foco.

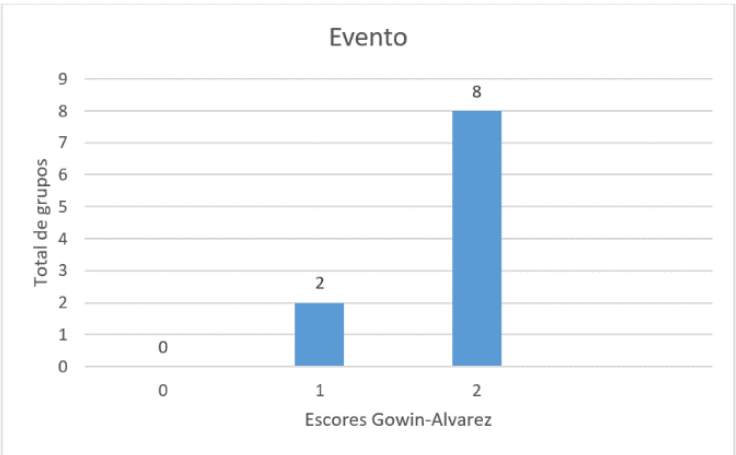

Gráfico 6: Evento nos diagramas Fonte: Os autores, 2018
Em um trabalho o item Registros/Dados (Gráfico 7) não foi identificado. Quatro grupos apresentaram Registros/Dados, mas inconsistentes com a Questão-foco ou com o Evento. Cinco grupos foram coerentes com este item ao relacioná-lo ao Evento e a Questão-Foco. Os elementos constitutivos do Diagrama $\checkmark$ funcionam de forma normativa para estabelecer os critérios de valor. Uma boa porção de conhecimento deverá incluir todos os elementos do "Vê", ilustrar como é que esses elementos se ligam entre si, e ser coerente, compreensiva e significativa [8].

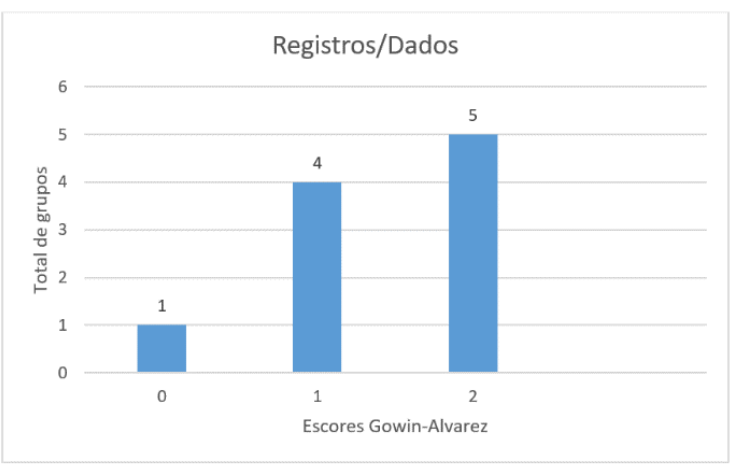

Gráfico 7: Registro/Dados nos diagramas Fonte: Os autores, 2018

Podemos observar no Gráfico 8, as Transformações apresentaram bom desempenho para sete grupos, pois estava em consonância com a Questão-Foco. Três grupos não tiveram a mesma facilidade. Registros muito incompleto e as Transformações incoerentes com a Questão-Foco.

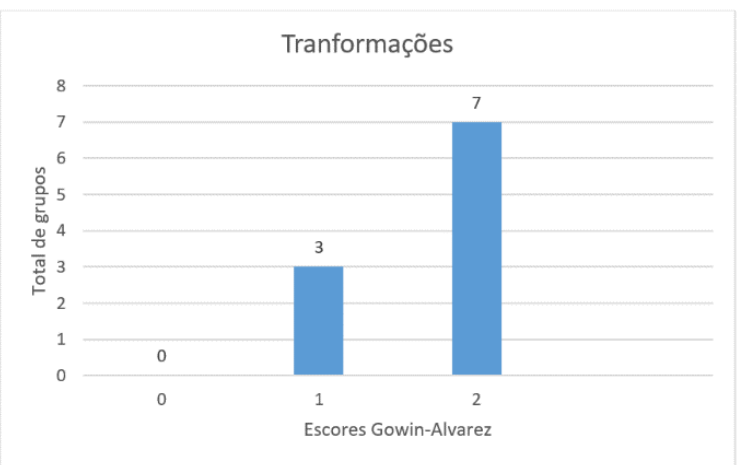

Gráfico 8: Transformações nos diagramas Fonte: Os autores, 2018

O item Conclusões e Justificativas (Gráfico 9), traz resultado satisfatório, apesar que um dos grupos, não apresentou a conclusão do trabalho. Outro grupo apresenta Conclusões, mas inconsistentes com a Questão-foco. Dois grupos apresentam Conclusões, derivadas dos Registros e Transformações. Quatro grupos apresentam Conclusões consistentes com os 


\section{Blucher Proceedings \\ X Encontro Científico de Física Aplicada}

dados coletados nos Registros e representados nas Transformações. Dois grupos trazem as Conclusões, que contêm os componentes com os dados coletados nos Registros e representados nas Transformações e sugerem novas Questão(ões)-foco.

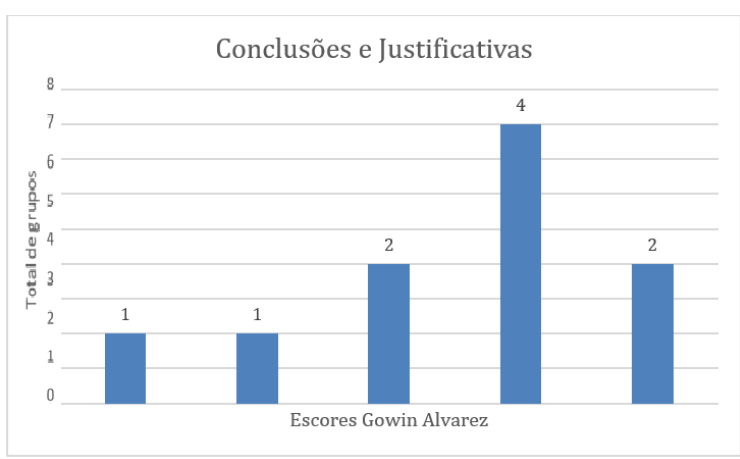

Gráfico 9: Conclusões e Justificativas nos diagramas Fonte: Os autores, 2018

O item o Resultado encontrado coincide com o que você esperava? Um grupo não apresentou nenhum Resultado (Gráfico 10). Três grupos mostraram seus Resultados, mas não está relacionado com a QuestãoFoco e/ou o Evento. Para os demais grupos foram, o Resultado é identificado e está relacionado com a Questão-Foco e/ou o Evento.

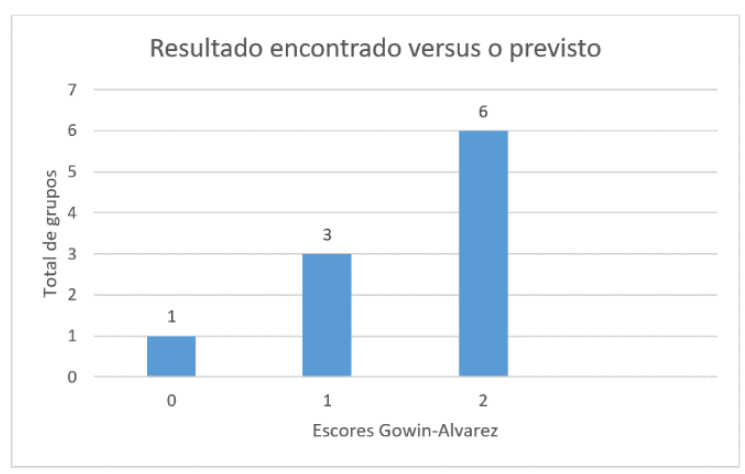

Gráfico 10: Resultado encontrado versus o esperado Fonte: Os autores, 2018

Com relação ao elemento do Diagrama V, Questão-Foco, os alunos não têm o hábito em seus experimentos para a Feira de Ciências partir de um problema (Porque?). O evento científico da escola precisa passar por reformulação e trazer a linguagem científica para os educandos. O Diagrama V (Figura 2), como método de pesquisa, é necessário bom planejamento, boa técnica, amostra, instrumento de coleta de dados e critérios de análise.

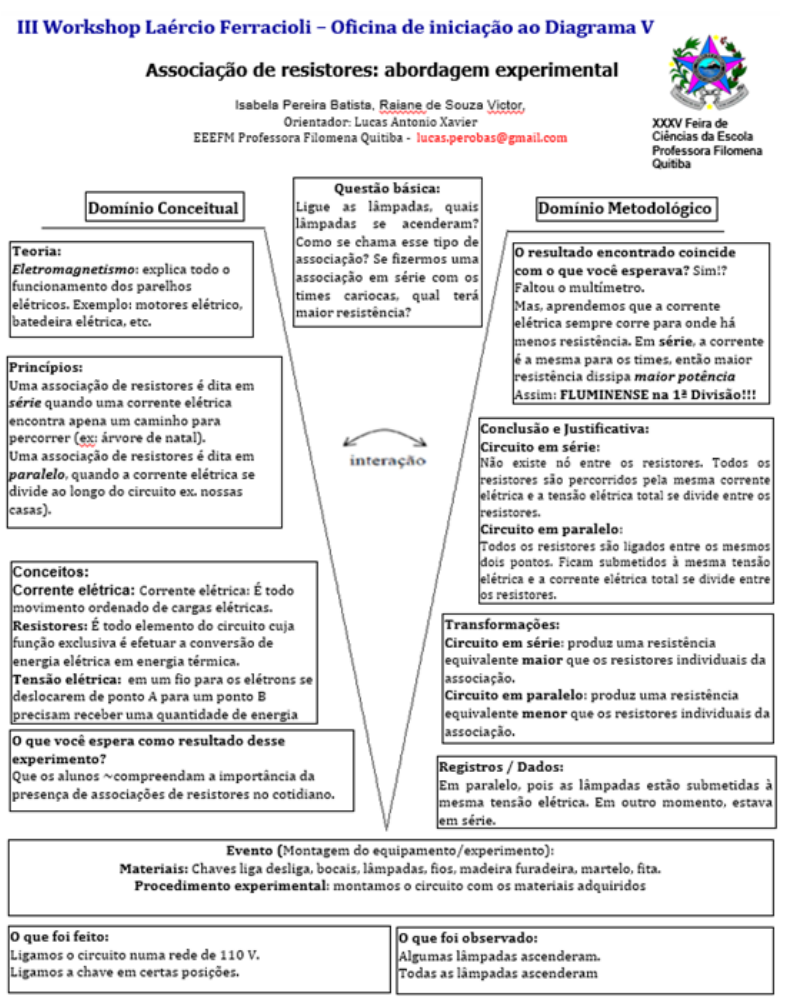

Figura 2: Diagrama $\mathrm{V}$ do grupo Associação de resistores: abordagem experimental.

Fonte: Os autores, 2018

A infusão do Diagrama $V$ nas atividades experimentais para a Feira de Ciências teve o propósito de facilitar a construção do conhecimento científicos contidos nos experimentos.

\section{Conclusão}

Para efeito de conclusão, o uso do Diagrama $\checkmark$ como recurso instrucional adotado no evento científico promovido anuamente pela escola como alternativa foi assertivo ao promover capacitação dos estudantes por meio de oficinas pedagógicas.

Com os experimentos concretizados e Diagrama $\mathrm{V}$ devidamente preenchido pelos membros dos grupos, pôde-se perceber que eles se sentiram mais confiantes e com argumentos plausíveis da atividade realizada. Com relação ao preenchimento do diagrama, eles tiveram dificuldade com o lado direito (Domínio Metodológico) e de relacionar os elementos com a Questão-Foco ou Evento.

As oficinas realizadas a fim de explorar o método científico à luz do Diagrama $V$, se mostraram estratégica. Os integrantes dos grupos concordaram com o recurso heurístico e querem a sua implementação 
no evento da escola. Pois, ajuda a identificar os componentes do conhecimento, clarificar suas relações e apresentá-los de modo claro e visualmente compacto [9].

As implicações teóricas deste trabalho apontam para caminhos a serem percorridos no projeto Feira de Ciências para potencializar a aprendizagem dos educandos e capacitá-los a seguirem no campo da ciência. $E$, para os próximos trabalhos discutir o Diagrama $\mathrm{V}$, infundir em outras tarefas escolares, com a finalidade de aproveitar as potencialidades que ele traz agregando conhecimento para o educando.

\section{Referências}

[1] GOWIN, D.B. Educating. Ithaca, Cornel University Press, 1981.

[2] GOWIN, D. B.; ALVAREZ, M. C. The Art Educating with V Diagrams. Cambridge University Press, New York, 2005.

[3] BRASIL. Ministério da Educação. Secretaria de Educação Básica. Programa Nacional de Apoio às Feiras de Ciências da Educação Básica. Fenaceb. Brasília, DF, 2006.

[4] PRADO, R. T. Utilização do diagrama v em atividades experimentais de física em sala de aula de ensino médio. Vitória, 137 p., 2015. Dissertação (Mestrado) - Universidade Federal do Estado do Espírito Santo.

[5] MOREIRA, M. A. Diagramas V e Aprendizagem Significativa. Revista Chilena de Educación Científica, vol. 6, N. 2, pp. 3-12. 2007. Revisado em 2012.

[6] BRASIL. Diretrizes Curriculares Nacionais Gerais da Educação Básica/ Ministério da Educação. Secretária de Educação Básica. Diretoria de Currículos e Educação Integral. - Brasília: MEC, SEB, DICEI, 2013.

[7] CORSINI, A.M.A.; ARAÚJO, E.S.N.N. Feira de Ciências como espaço não formal de ensino: um estudo com alunos e professores do ensino fundamental. In: VI ENPEC Encontro Nacional em Pesquisa e Educação para Ciências, 2007, Florianópolis-SC.

[8] NOVAK, J. D.; GOWIN, D. B. Aprender a Aprender. Lisboa: Plátano Edições Técnicas, 1984.

[9] CAPPELLETTO, E. O Vê de Gowin conectando teoria e experimentação em física geral: questões didáticas, metodológicas e epistemológicas relevantes ao processo. Porto Alegre, 297 p., 2009. Dissertação (Mestrado) - Universidade Federal do Rio Grande do Sul. 